\title{
POSITRON EMULATOR FOR COMMISIONING ILC POSITRON SOURCE
}

\author{
H. Wang, W. Liu, W. Gai, and K-J. Kim, ANL, Argonne, IL 60439, U.S.A.
}

\section{Abstract}

The gamma-ray based positron source [1] is an attractive option for the International Linear Collider (ILC) positron option because of the less stringent target requirement and the possibility to provide polarization. The drawback is the fact that the positron accelerator system including positron linac and damping ring can not be fully commissioned until the electron accelerator system is commissioned at high energies $(>150 \mathrm{GeV})$. In this paper, we discuss a scheme that would use a low energy $(\sim 100 \mathrm{MeV})$ electron beam scattered through a set of carefully selected targets to mimic phase space distribution of the positron beam. The positron "emulator" can be used to effectively commission the positron accelerator system including the capture optics, the linac, and even the damping ring before the ILC colliding electron beam is ready. Simulation results using EGS4 for beam scattering and PARMELA for beam dynamics are presented.

\section{INTRODUCTION}

The ILC requires a high intensity positron source that can be achieved using gamma ray based scheme [1-3]. In this scheme, a high energy electron beam passes through a helical undulator produces high energy photons (gamma rays), and then the photons strike a thin Ti-target (0.4 X0) to create electron and positron pairs. A benefit of this scheme is that one can obtain longitudinally polarized positrons.

In this paper, we follow the TESLA design [2] but with a lower electron beam energy of $150 \mathrm{GeV}$ to produce gamma rays [4]. The initial distribution of particles (positron and electron) emerging from the conversion target is produced using the electromagnetic shower simulation code, EGS4 [5]. Then, the generated positrons and electrons go into the positron capture optics, which consists of the Adiabatic Matching Device (AMD) [1] and the pre-accelerator. The AMD, a tapered solenoid with a high initial B field and tapering adiabatically down to the constant end field, can match initially large divergence particles into the aperture of pre-accelerator. Next, the pre-accelerator accelerates the particles to $\sim 120 \mathrm{MeV}$. At this point, the positrons satisfying the constraint of damping ring acceptance are captured, passed through quadrupole focusing system, and continuously accelerated up to $5 \mathrm{GeV}$ before being transported to damping ring. We have performed a complete study of beam dynamics (including electron and positron) from conversion target to the entrance of damping ring using particle tracking code, PARMELA [6].

In addition to the simulations of the gamma ray based positron source, we also consider a positron emulator. A low energy electron beam $(\sim 100 \mathrm{MeV})$ from an L-band $\mathrm{RF}$ photoinjector is scattered by a target designed so that the scattered electrons have the same distribution as the real positron source. We suggest that this "emulated" positron beam can be used to commission positron capture optics, boosting linacs, and damping ring without needing to wait for the $150 \mathrm{GeV}$ electron beam to be commissioned.

\section{BEAM DYNAMICS STUDIES OF GAMMA RAY BASED POSITRON SOURCE}

In this section, we present a simulation of the gamma ray based positron source using EGS4 and PARMELA Our simulations include the positron generation, capture, and acceleration up to $5 \mathrm{GeV}$, but not the gamma ray source (the undulator) itself.

\section{Positron Generation}

The parameters for the undulator used to produce the gamma rays and positron target are taken from [3] and listed at Table 1.

Table 1: Parameters for the undulator and positron target

\begin{tabular}{|l|l|}
\hline \multicolumn{2}{|l|}{ Helical undulator } \\
\hline Drive electron beam energy & $150 \mathrm{GeV}$ \\
\hline $\mathrm{K}$ & 1.5 \\
\hline Magnetic field,B & $1.76 \mathrm{Tesla}$ \\
\hline Target parameters & $\mathrm{Ti}$ \\
\hline Target material & $1.42 \mathrm{~cm}(0.4 \mathrm{Xo})$ \\
\hline Target thickness
\end{tabular}

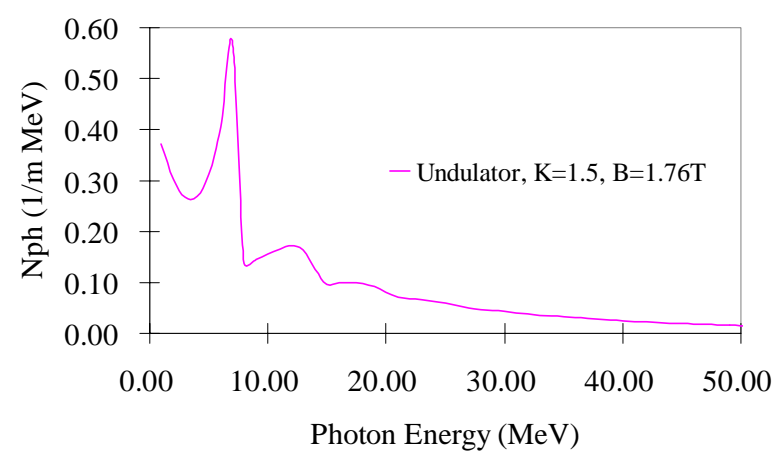

Figure 1: Photon energy spectrum generated by helical undulator using $150 \mathrm{GeV}$ electron beam.

Using Eqn. 1.8 from [1], we plot the photon energy spectrum generated in the undulator using a $150 \mathrm{GeV}$ electron beam in Figure 1. Figure 2 shows the initial positron energy spectrum produced using EGS4. 


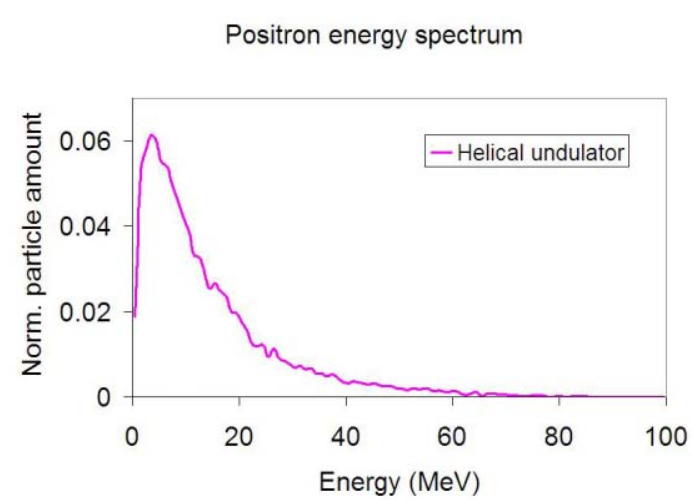

Figure 2: The initial positron energy spectrum. This is the spectrum at the exit of the target.

\section{Positron Capture Optics}

The positron capture optics consists of a tapered solenoid (the AMD) and 15 linac tanks (the preaccelerator) embedded in a solenoidal field. The magnetic field at the beginning of the AMD is 5 Tesla and it tapers down adiabatically to 0.25 Tesla over its $50 \mathrm{~cm}$ length. The pre-accelerator includes fifteen, 5-cell, $\pi$ mode, L-band linacs, immersed in a constant solenoidal field of $\mathrm{B}=0.25$ Tesla. Each linac has an accelerating gradient $12 \mathrm{MV} / \mathrm{m}$ with an aperture radius equal to $25 \mathrm{~mm}$.

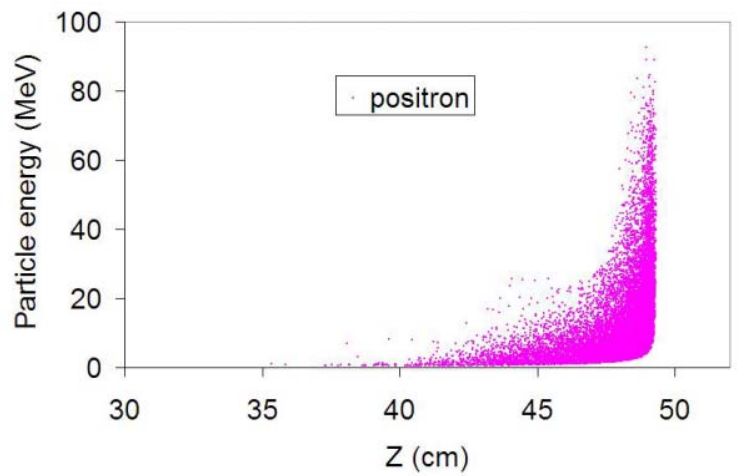

Figure 3: Longitudinal phase space at the time the reference particle exits the AMD.

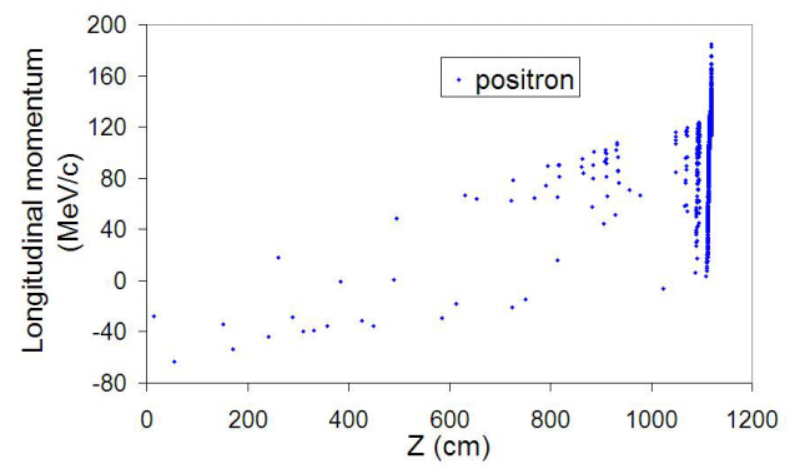

Figure 4: A snapshot of longitudinal momentum distribution at the time the reference particle exits the preaccelerator.
The initial distribution of the particles emerging from target is generated with EGS4. The distribution parameters are: spot radius of $2 \mathrm{~mm}$; divergence $(\mathrm{Px} / \mathrm{P})$ of up to $1 \mathrm{rad}$ in transverse phase space; bunch length of $8 \mathrm{ps}$; and energy spread from 0 to $100 \mathrm{MeV}$. Due to this large initial transverse momentum spread, the path lengths of the positrons in the AMD vary significantly and the beam bunch length increases significantly (Fig. 3). The positrons and electrons are separated in the preaccelerator where each species is captured by accelerating buckets that differ in phase by half an RF wavelength. Figure 4 gives a snapshot of the longitudinal momentum distribution of the positrons. Since a standing wave linac is used to accelerate particles, some particles are decelerated and finally accelerated at counter direction.

\section{Capture Efficiency at the Pre-accelerator Exit}

The damping ring acceptance for positron capture is: energy spread $\leq \pm 0.5 \%$ (corresponding to $15^{\circ} \mathrm{RF}$ phase) and normalized transverse emittance $\varepsilon x+\varepsilon y \leq 0.048 m \cdot r a d$. Based on this capture window, we calculate the capture efficiency, $\eta=N_{c} / N_{t}$, where $\mathrm{N}_{\mathrm{c}}$ is the number of positrons in the capture window at the exit of the pre-accelerator and $\mathrm{N}_{\mathrm{t}}$ is the total number of positrons emerging from target. Figure 5 shows the captured positrons at pre-accelerator exit. The capture efficiency is $27 \%$, and the normalized r.m.s. transverse emittance of the captured positrons is $0.0042 \mathrm{~m} \cdot \mathrm{rad}$.

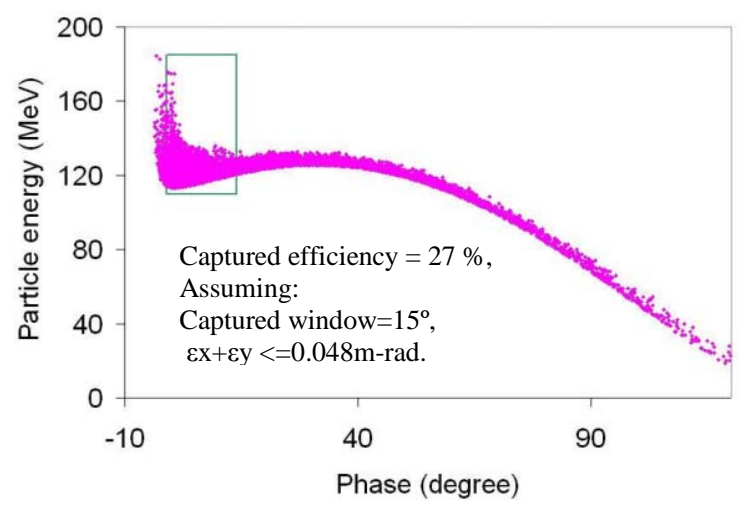

Figure 5: Capture efficiency at pre-accelerator exit. Captured positrons are located in the captured window.

A helical undulator can produce circularly polarized gamma rays, which hit a target to generate longitudinally polarized positrons [3]. The polarization of the captured positrons is obtained using EGS4 according to the approach described in [1]. About half of the captured positrons have a polarization $>0.7$ and the average polarization of all the positron is 0.6. This meets the polarization requirements for the ILC.

PARMELA simulation shows that about $70 \%$ of the electrons and $50 \%$ of the positrons are lost at the AMD and the pre-accelerator. This creates a radiation hazard. Assuming $3 \mathrm{nC}$ for the captured positrons and $3 \mathrm{nC}$ for the driving electrons at $150 \mathrm{GeV}$, we calculate the power of the total gamma rays, the lost particles, and the power 
deposited at conversion target by backfired particles in Table 2 .

Table 2: Some results under the constraint of different damping ring acceptance.

\begin{tabular}{|l|l|l|}
\hline $\begin{array}{l}\text { Damping ring } \\
\text { constraint }\end{array}$ & $\begin{array}{l}\varepsilon \mathrm{x}+\varepsilon \mathrm{y}<= \\
0.048 \mathrm{~m} \cdot \mathrm{rad}\end{array}$ & $\begin{array}{l}\varepsilon \mathrm{x}+\varepsilon \mathrm{y}<= \\
0.024 \mathrm{~m} \cdot \mathrm{rad}\end{array}$ \\
\hline Gamma ray power & $67 \mathrm{KW}$ & $80 \mathrm{KW}$ \\
\hline Power of lost particles & $16 \mathrm{KW}$ & $20.2 \mathrm{KW}$ \\
\hline $\begin{array}{l}\text { Power deposited in } \\
\text { target by backfired } \\
\text { particles }\end{array}$ & & \\
\hline
\end{tabular}

At the pre-accelerator exit, the positrons meeting the damping ring acceptance must be separated from background particles to reduce the power consumption in the subsequent accelerating stage. A positron separation device to do this is still under study. In the simulation, the captured positrons are artificially separated, and further accelerated to $5 \mathrm{GeV}$ using about 200 TESLA-type 9-cell $\pi$-mode L-band superconducting linacs. Quadrapole doublets are used to focus the captured positron beam. During the acceleration process, no particle is lost. The output has recently been used as the input for the ILC damping ring design. [7]

\section{POSITRON EMULATOR STUDIES}

The gamma ray based positron source requires a high energy driving electron beam $(>150 \mathrm{GeV})$. This will delay the commissioning process of the positron capture optics, boosting linacs, and damping ring. The purpose of the positron emulator studies is to find a scheme to condition these positron systems without the real positron source.

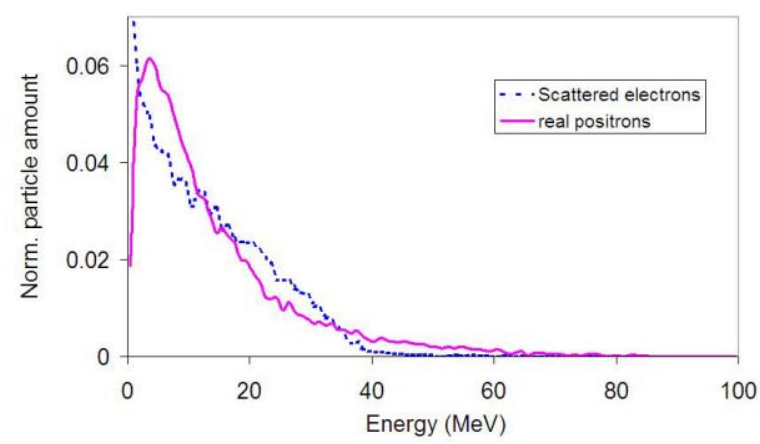

Figure 6: Energy distribution. Comparison of the energy spectrum at target exit for the two sources.

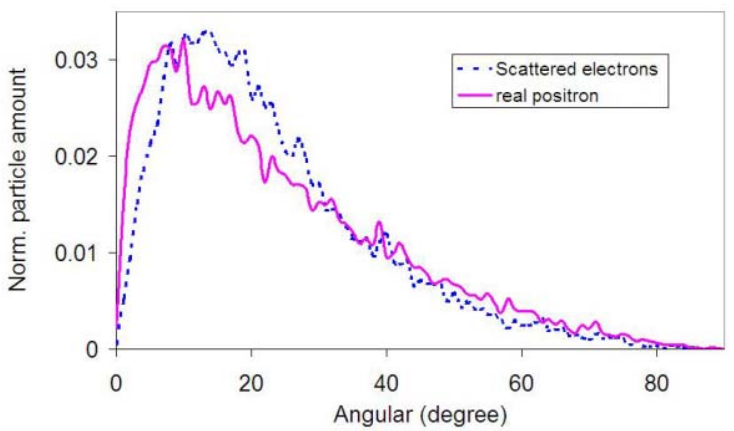

Figure 7: Angular distribution. Comparison of the energy spectrum at target exit for the two sources.

The idea behind the emulator is to mimic certain aspects of the real positron source with a low energy electron beam. However, since the electron beam generated by the RF photoinjector has a low emittance, a target is selected to produce a scattered beam that mimic the positron phase space. We simulated targets of different material and thickness and different incident beam energy. Using a Beryllium target of $30 \mathrm{~cm}$, and incident electron beam energy of $120 \mathrm{MeV}$, the emulator and real distributions are shown to be good agreement in Figures 6 and 7.

\section{CONCLUSION}

Simulations for both the electron and positron have been studied from the conversion target to the damping ring input using PARMELA. A $27 \%$ positron capture efficiency was obtained with the constraint of damping ring acceptance. The polarization of the captured positrons is $60 \%$, which meets the ILC requirement.

By choosing the right target and incident electron beam, simulations show that we can "emulate" the distribution of the real positron source. Using this positron emulator, the positron capture optics, boosting linacs, and damping ring can be conditioned long before the real positron source is available.

\section{REFERENCES}

[1] K. Flöttmann, PhD Dissertation, DESY 93-161, Hamburg 1993.

[2] TESLA Technical Design Report, DESY 2001-011.

[3] V.E. Balakin, A.A. Mikhailichenko, Prepint: INP 79$85,1979$.

[4] U.S. Linear Collider Technology Options Study, 2004.

[5] W. Nelson, H. Hirayama, D. Rogers, .The EGS4 code system. SLAC report 265, 1985.

[6] L.M. Young (documentation by J.H. Billen). "PARMELA", report LA-UR-96-1835, Los Alamos, 1996 (rev. 2004).

[7] A. Xiao, private communications 